\title{
An inter-professional approach towards understanding the science, myths and realities of COVID-19 pandemic
}

\author{
L.M. Azzopardi*, A. Serracino-Inglott \\ Department of Pharmacy, Faculty of Medicine and Surgery, University of Malta, Malta
}

\author{
Keywords \\ E-learning \\ Communication \\ Inter-professional Education \\ COVID-19 \\ Malta \\ *Corresponding author \\ lilian.m.azzopardi@um.edu.mt
}

\begin{abstract}
Summary: As it spread to pandemic levels, COVID-19 viral infection presented a number of challenges for education, health systems and the wellbeing of society. Evidence-based information on COVID-19 was essential for designing the response to the pandemic. A series of weekly webinars were developed and presented over a 15week period. An inter-professional panel of experts contributed to the discussions during the webinars. The webinars were planned through a multidimensional approach involving students and practitioners from different healthcare professions encompassing a range of scientific aspects from basic sciences to socio-economic aspects. The evaluations showed the value of the webinars as an e-learning method for inter-professional communication in the discussion and analysis of the scientific evidence and sharing of experiences and data.
\end{abstract}

\section{Background and Context}

With the mitigation strategies adopted on university campuses to halt the spread of COVID-19, pharmacy education activities were abruptly redesigned to adopt remote learning platforms to replace live sessions, practical sessions and placements. Remote learning reduced informal contact of faculty and students within healthcare professions that often formed the basis for an inter-professional approach (Sorensen, Lin, \& Allen, 2020). The pandemic accelerated an evolution in pharmacy education, particularly the implementation of digitalisation in teaching and assessment activities (Lyons, Chrisopoulos, \& Brock, 2020). Publications about the aspects of COVID-19 were being published at an accelerated speed with the pre-print platforms putting forward early access to study results (Glasziou, \& Sanders, 2020). Due to the inclusion of the School of Pharmacy in the Faculty of Medicine and Surgery at the University of Malta, the Department of Pharmacy has always worked on amalgamating education, practice, and research to provide synergy across healthcare professions in the advancements in education and practice. This was the Department's driver in the development of an e-learning model based on an inter-professional approach towards understanding the science produced during the COVID-19 pandemic (Azzopardi \& Serracino-Inglott, 2020). The model developed was multi-dimensional and sought to support both students and practitioners from different healthcare professions in the discussion and analysis of the scientific aspects of the pandemic, enabling critical analysis of the literature from different points of view including clinicians, pathologists, pharmacists, public health officers, epidemiologists and biostatisticians. The context of the model was based on the relevance of multi-stakeholder networking in a global milieu within pharmacy education within an international perspective (Anderson et al., 2009).

\section{Educational Description}

The objective of the model was to provide an e-learning experience which presented scientific evidence and reflections on the challenges of interpreting the science, myths and realities of COVID-19 pandemic. A series of webinars intended to tackle the perceived needs were developed; these were addressing the knowledge gap 
expressed by healthcare professionals; maintaining interprofessional communication; spurring the application of basic science to mitigation strategies; and patient care. The webinar design was led by an expert panel of interprofessional educators who decided collectively on the content and timeliness of the information shared.

A series of 15 webinars were prepared and presented individually on a weekly basis, each had a duration of 45 minutes with 15 minutes discussion. The webinars were led by an academic pharmacist with participation from a panel of experts including virologists, immunologists, specialists in infectious disease, internal medicine and intensive care, bioethicists, epidemiologists and pharmaceutical and public health regulators. In order to promote interactivity and discussion during the webinars, participation was limited to 100 participants per session. Each webinar was repeated (three to five times) as a live session to accommodate the registrations.

The webinar topics covered the presentation of the infection; the rationale for re-positioning medicines; news on the ongoing clinical trials; evidence of different treatment strategies; medical devices; use of personal protective equipment; scientific-evidence related to containment measures; laboratory investigations and interpretation; dynamics of predisposition to the disease and vulnerable persons; development of vaccines; CRISPR-RNA technologies in antiviral defence; the consequences of lockdown (affordability, social health and wellbeing); correlations and causality; improvisation and optimisation in handling a pandemic; and risk policies and strategy developments.

The 250 webinar participants were a mixture of health professionals including students and academics from international academic institutions. The webinars were offered as an elective experience and a certificate of attendance was issued to participants. The webinars were scheduled in the evenings so as to facilitate the participation of practitioners. At the end of the series of webinars, an anonymous online survey was disseminated to all participants to evaluate the sessions by rating the relevance of the topics addressed, content and the learning experience. Direct qualitative feedback was sought by seeking responses from a convenience sample of participants and panel members.

\section{Outcomes and Recommendations}

The feedback received identified the value of the webinars as a learning experience where a concise analysis of the scientific evidence was put forward and a platform for discussions and sharing of experiences and data was provided.

Fifty responses (33 female, 16 male, collective mean age of 32 years, age range 20-66 years) were received for the evaluation. The respondents were pharmacists (15), students (22), physicians (5), scientists (4), pharmacy administrators and patient advocates (4). Feedback indicated that the topics addressed were extremely or highly relevant (46), always informative (33) and the sessions exceeded the expectations of 27 participants. Clarity, organisation and scientific content of the webinars were rated as 'high' to 'very high' by the majority of the participants (45). The contributions by the inter-professional panel were rated as 'extremely relevant' and 'highly relevant' by 46 respondents. The three topics most highly ranked were:

1. Public health containment measures

2. Vaccine development

3. Testing and interpretation.

The topics that were least ranked were CRISPR RNA in antiviral defence, strategy and risk policies, and improvisation and optimisation in pandemics.

The objectives included the provision of an e-learning activity within an inter-professional dimension to mobilise communication and addressing the challenges brought forward by the COVID-19 pandemic. Therefore, the outcome of this innovative teaching and learning model was the overall achievement of the objectives. The model provided an opportunity to highlight the significance of lifelong learning skills as it was a voluntary learning exercise with scope to spur learning and sharing in challenging and unexpected times.

A recommendation from this case study is the need and value within pharmacy education for schools of pharmacy to lead in initiatives which go beyond addressing curricular needs, and focus on supporting collaborative practices and analysis of how science applies to practice transformation.

\section{References}

Anderson, C., Bates, I., Beck, D., Brock, T.P., Futter, B., Mercer, H., Rouse, M., Whitmarsh, S., Wuliji, T., \& Yonemura, A. (2009). The WHO UNESCO FIP pharmacy education taskforce. Human Resources for Health, 45(7). https://doi.org/10.1186/1478-4491-7-45

Azzopardi, L.M., \& Serracino-Inglott A. (2020). Clinical pharmacy education and practice evolvement in Malta. Journal of the American College of Clinical Pharmacy, 1-7. https://doi.org/10.1002/jac5.1280

Glasziou, P.P., \& Sanders, S. (2020) Waste in covid-19 research. British Medical Journal, 369. https://doi.org/10.1136/bmj.m1847

Lyons, K.M., Chrisopoulos, A., \& Brock, T.P. (2020). Sustainable pharmacy education in the time of COVID-19. American Journal of Pharmaceutical Education, 84(6), Article 8088. https://doi.org/ $\underline{10.5688 / a j p e 8088}$

Sorensen, T.D., Lin, A., \& Allen, D.D. (2020). Reinventing how pharmacy educators connect as a community. American Journal of Pharmaceutical Education, 84(6), Art. 8151. https://doi.org/10.5688/ aipe8151 